\title{
Determinação de idade e crescimento do mapará (Hypopbthalmus marginatus) na Amazônia Central.
}

\author{
Leocy CUTRIM ${ }^{1}$, Vandick da Silva BATISTA ${ }^{2}$
}

\begin{abstract}
RESUMO
O presente trabalho visa contribuir para a conservação e manejo do mapará (Hypophthalmus marginatus), um importante recurso pesqueiro de exportação do Amazonas para o qual é necessário conhecer informações essenciais de sua dinâmica de populações. Para contribuir à esta finalidade, o presente trabalho estabeleceu como objetivo identificar o par de otólito mais adequado para leitura de anéis etários, determinar os parâmetros de crescimento do mapará (H. marginatus), Amazônia Central, Amazonas - Brasil. O trabalho foi efetuado através da análise de otólitos coletados no período de dezembro de 1996 á agosto de 1997. Os otólitos escolhidos foram os asteriscus, sendo a leitura efetuada em lupa estereoscópica com monitor acoplado. As marcas de crescimento foram validadas por meio da análise do incremento marginal relativo, sendo encontrado dois anéis/ano. Os valores estimados para os parâmetros no período foram $\mathrm{L} \infty=52,63 \mathrm{~cm} ; \mathrm{k}=0,555$ ano; to $=-0,029 \mathrm{e} \mathrm{M}=0,552$. O ciclo hidrológico e comportamento reprodutivo estão relacionados com a marcação de anéis etários.
\end{abstract}

\section{PALAVRAS-CHAVE}

Idade, crescimento, Hypophthalmidae, Amazônia.

\section{Age and growth of mapará (Hypophthalmus marginatus) in the Central Amazon.}

\begin{abstract}
The present work seeks to contribute for the conservation and management of the mapará(Hypophthalmus marginatus), an important export fishing resource of Amazonas for which is necessary to know essential information of itspopulation dynamics. To contribute to this purpose, the present work established as objective to identify a structure for reading of age rings, to determine the parameters of growth of the mapará (H. marginatus), Central Amazon, Amazonas - Brazil. The work was made through the otoliths analysis collected in the period of December of 1996 to August of 1997. The otolith chosen for reading was the asteriscus, being the reading made in a stereomicroscope with coupled monitor. The mark structures were validated by means of the analysis of the relative marginal increment, being found two rings. The values estimated for the parameters in the period were $L \propto=52,63 \mathrm{~cm} ; k=0,555$ year; $t o=-0,029$ and $M=0,552$. The bydrologic cycle and reproductive behavior were the responsible factors for age rings.
\end{abstract}

\section{KEYWORDS}

Age, growth, Hypophthalmidae, Amazon

\section{INTRODUÇÃO}

Os maparás (Hypophthalmus spp.) são Siluriformes de porte médio, pertencentes a família sul-americana Pimelodidae com um gênero e três espécies (H. marginatus, H. edentatus e H. fimbriatus). São peixes reoflicos, pois dependem da correnteza do ambiente natural para realizar suas funções reprodutivas (Carvalho, 1980a). Diferem dos Siluriformes em geral, quanto ao hábito alimentar e localização na coluna d'água, pois enquanto a maioria é demersal e carnívoro, os maparás são estritamente pelágicos e planctófagos. Alcântara Neto (1994) e Carvalho (1980a), realizaram estudos sobre a alimentação do mapará, verificando que os principais tipos de alimentos que tomam parte na sua dieta são crustáceos planctônicos. A distribuição dos maparás é ampla na América do Sul; incluindo bacia amazônica, do Prata, Orinoco e águas costeiras do Pará (foz do Amazonas) até o Suriname. Na Amazônia Central são encontradas as três espécies do gênero Hypophthalmus (Alcântara-Neto, 1994).

\footnotetext{
${ }^{1}$ Engenheiro de Pesca, Bolsista CNPq/PIBIC durante o trabalho. E-mail: leocy@inpa.gov.br.

${ }^{2}$ Professor do Departamento de Ciências Pesqueiras - UFAM - Manaus/AM, 69077-000 E-mail: vbatista@fua.br
} 
Carvalho (1980b), estudando a reprodução do H. edentatus no lago do Castanho, constatou que a desova do mesmo ocorreu quando as águas atingiram seu nível mais elevado..

O mapará é de interesse econômico, porém, da mesma forma que outros peixes lisos o consumo no Amazonas é baixo, devido a tabus alimentares, sendo a maioria da produção comercializada para outros países e estados do Brasil. As pescarias comerciais destes peixes na região ocorrem principalmente nos rios Amazonas, Negro, Solimões próximo das cidades de Codajás, Coari, Itacoatiara, Beruri, Manacapuru e Iranduba (Batista, com. pess.). Segundo Alcântara Neto (1994), no lago Grande de Monte Alegre a safra do mapará coincide com a enchente. Na Amazônia Central, a safra do mapará ocorre entre os meses de fevereiro a agosto, época de cheia na região (Batista, com pess.).

Uma avaliação realizada no Baixo Tocantins, antes do fechamento da barragem da hidroelétrica de Tucuruí, indicou que o mapará (H. marginatus) estava sendo explorado em um nível muito alto (Merona, 1993)

Considerando a crescente pressão de pesca sobre os estoques de $H$ marginatus, com possíveis conseqüências ecológicas e econômicas para a região, tornam-se necessários estudos sobre sua dinâmica populacional. Dentre os estudos fundamentais, está a determinaçãoda idade e estimativa dos parâmetros de crescimento do estoque, assim como a avaliação de fatores bióticos e abióticos que influenciam no crescimento da população ao longo do ano.

Ainterpretação de marcas anuais em estruturas calcificadaséo método mais aceito para a determinação da idade em peixes (Casselman, 1983). Tais determinações podem ser efetuadas em várias estruturas rígidas nos peixes: escamas, otólitos, vértebras, ossos operculares, espinhos das nadadeiras dorsal e peitoral entre outras. Para os Siluriformes, os otólitos, as vértebras e os espinhos peitorais e odorsal, são as estruturas mais frequentemente utilizadas para determinaçãode idade, jáque os mesmos nãopossuemescamas que possam evidenciar anéis etários. (Panfili, 1993)

O presente trabalho tem como objetivos identificar os otólitos mais adequados para a determinação de idade de $H$. marginatus; efetuar a validação das marcas de crescimento e estimar a curva de crescimento para a espécie para subsidiar o manejo da pesca do mapará na Amazônia Central.

\section{MATERIAIS E MÉTODOS}

Para determinação da idade e crescimento do mapará ( $H$. marginatus), foram realizadas amostragens de peixes provenientes da pesca comercial, principalmente do Frigorífico Iranduba - Friuba, no município de Iranduba, Amazonas. O período de coletas foi de dezembro de 1996 a agosto de 1997.

Durante o desembarque foram obtidas informações sobre a espécie diretamente da tripulação dos barcos. As amostras procederam dos seguintes locais: lago doJanauacá e do município de Manaquiri. Após a coleta, os peixes foram transportados ao Laboratório de Avaliação e Manejo da Pesca-UFAM, para obtenção de dados biológicos . Foram registrados os seguintes dados de cada exemplar: comprimento furcal $(\mathrm{mm})$; comprimento padrão (mm); peso eviscerado (g) e sexo através de observação macroscópica das gônadas. Em seguida, foram retirados os 3 pares de otólitos: Lapillus (maior), Asteriscus (médio) e o Sagitta (menor). Após a remoção dos arcos branquiais, foram retirados os otólitos, situados na porção da cabeça situada na cápsula auditiva, sendo retirados dos sáculos que os envolviam, e lavados em água corrente e preservados com álcool $70 \%$.

Na visualização, foi utilizado um microscópio estereoscópico com ocular micrométrica, conectado a um monitor colorido. A leitura foi efetuada com iluminação lateral incidente, estando os otólitos imersos em álcool $70 \%$ e observados sob aumento de 40 vezes. Foram realizadas 3 leituras independentes de 25 otólitos, para calibrar os critérios de interpretação das marcas. Os dados referentes a amostra (data, sexo e comprimento) e das leituras anteriores foram ignorados durante a leitura das estruturas, de acordo com os princípios dos experimentos duplamente cegos (Vieira \& Hoffmann, 1989).

Uma sub-amostra com 5 exemplares por classes de comprimento foi obtida para caracterizar o tipo de marca em cada estrutura calcificada, com objetivo de selecionar qual delasé melhor para a determinação da idade. Para tal foram considerados os seguintes critérios: claridade dos anéis, padrão de formação, presença de anéis intermediários e duplicidade de anéis.

Para a análise quantitativa dos anéis dos otólitos, foi escolhido o melhor ângulo de medida, determinado através do ajuste linear entre o raio da estrutura (com variação do ângulo de $0^{\circ} \mathrm{a}$ $45^{\circ}$ ) com o comprimento furcal. Este ajuste foi analisado através da avaliação do coeficiente de correlação e análise dos resíduos.

$\mathrm{O}$ incremento marginal relativo (IMR), foi calculado utilizando o raio total das estruturas calcificadas e o raio das duas últimas marcas, segundo a fórmula:

$I M R=\left(R_{t}-R_{n}\right) /\left(R_{n}-R_{p}\right) \quad$ (Lai et al., 1987)
$R_{t}=0$ raio total da estrutura;
$R_{n}=a$ distância do foco da estrutura até a última marca;
$R_{p}=$ a distância do foco da estrutura até a penúltima marca
Para relacionar a formação das marcas de crescimento com eventos sazonais do ciclo de vida, os valores médios mensais do IMR foram relacionados com informações de reprodução da espécie e com as variações mensais no nível da água do rio.

\section{Estimação dos Parâmetros Populacionais}

Foi ajustado aos dados de comprimento e idade, o modelo de crescimento de Von Bertalanffy:

$$
\begin{aligned}
& \mathrm{Lt}=\mathrm{L}_{\infty} *\left[1-\mathrm{e}^{-\mathrm{k}\left(\mathrm{t}-\mathrm{t}_{\mathrm{o}}\right)}\right] \text { sendo, } \\
& \mathrm{Lt}=\text { comprimento do peixe na idade } \mathrm{t} \\
& \mathrm{L} \infty=\text { comprimento infinito teórico; } \\
& \mathrm{k}=\text { taxa de crescimento } \\
& \mathrm{t}=\text { idade } \\
& \mathrm{t}_{\mathrm{o}}=\text { comprimento teórico do peixe na idade zero. }
\end{aligned}
$$

Os parâmetros $k$ e $t$ foram estimados pelo ajuste não linear das variáveis, utilizando o método iterativo Simplex, sem fixar o numero de iterações nem o valor semente. 


\section{ACTA AMAZONICA}

O L $\infty$ foi estimado para o total dos peixes, de acordo com Pauly, (1983) sendo:

$\mathrm{L}_{\infty}=$ comprimento máximo observado $/ 0,95$

A esperança de vida foi calculada pelo método de Taylor (apud Sparre \& Venema, 1995), através da seguinte formula:

$\mathrm{A}_{0,95}=\mathrm{t}_{\mathrm{o}}+(2,996 / \mathrm{k})$

Onde: $\quad \mathrm{A}_{0,95}=$ esperança de vida

$\mathrm{t}_{\mathrm{o}}=$ idade teórica para o comprimento zero

$\mathrm{k}=$ taxa de crescimento

Amortalidade natural (M) foi estimada pelo método de Taylor (Sparre \& Venema, 1995), considerando primeiro a longevidade $\left(\mathrm{A}_{0,95}=\mathrm{t}_{\mathrm{o}}+2,996 / \mathrm{k}\right)$; posteriormente, o parâmetro sendo:

$\mathbf{M}=-\ln (1-0,95) / \mathrm{A}_{0,95}$

\section{RESULTADOS}

No total, foram coletados 268 peixes, capturados pela pesca comercial de malhadeiras com malhas de amplitude 45 e 50 $\mathrm{mm}$ entre nós opostos e com comprimento entre 50 a $100 \mathrm{~m}$. Do total foram identificados 42 machos, 73 fêmeas e houveram 153 com sexo indeterminado (Tabela 1):

\section{- Lapillus (Figura 1a)}

Os lapillus se apresentam em maior tamanho (4,2 × 2,2 mm aproximadamente) e peso. São assimétricos sendo a porção ventral de menor tamanho em relação à dorsal. A face interna é convexa, junto á qual há uma deposição diferenciada de cálcio, impossibilitando a visualização dos anéis de crescimento na face interna. No sulco externo deste otólito, evidenciam-se anéis translúcidos e opacos, quando as estruturas são observadas com luz refletida.

\section{- Sagitta (Figura 1b)}

Menor tamanho (aproximadamente 2,4x de comprimento), que o lapillus e asteriscus, alongados, comprimidos lateralmente com forma de espinho. São de difícil manuseio muito frágeis, pois se quebram com facilidade no momento da retirada dos mesmos.

\section{- Asteriscus (Figura. 1c)}

Os asteriscus são de tamanho médio em relação aos outros e arredondados (aproximadamente 2,4 mm de diâmetro). Tanto o sulco interno quanto a externo, são um pouco plano, mas só observam-se anéis concêntricos bem marcados no interno.

O par de otólitos mais adequado para leitura foi o asteriscus, com anéis mais nítidos e forma manuseável, por apresentar pouca espessura permitindo melhor penetração de luz é possível uma melhor identificação dos anéis hialinos. Nas três leituras realizadas, sendo observado que apesar de não ter sido necessário utilizar nenhum
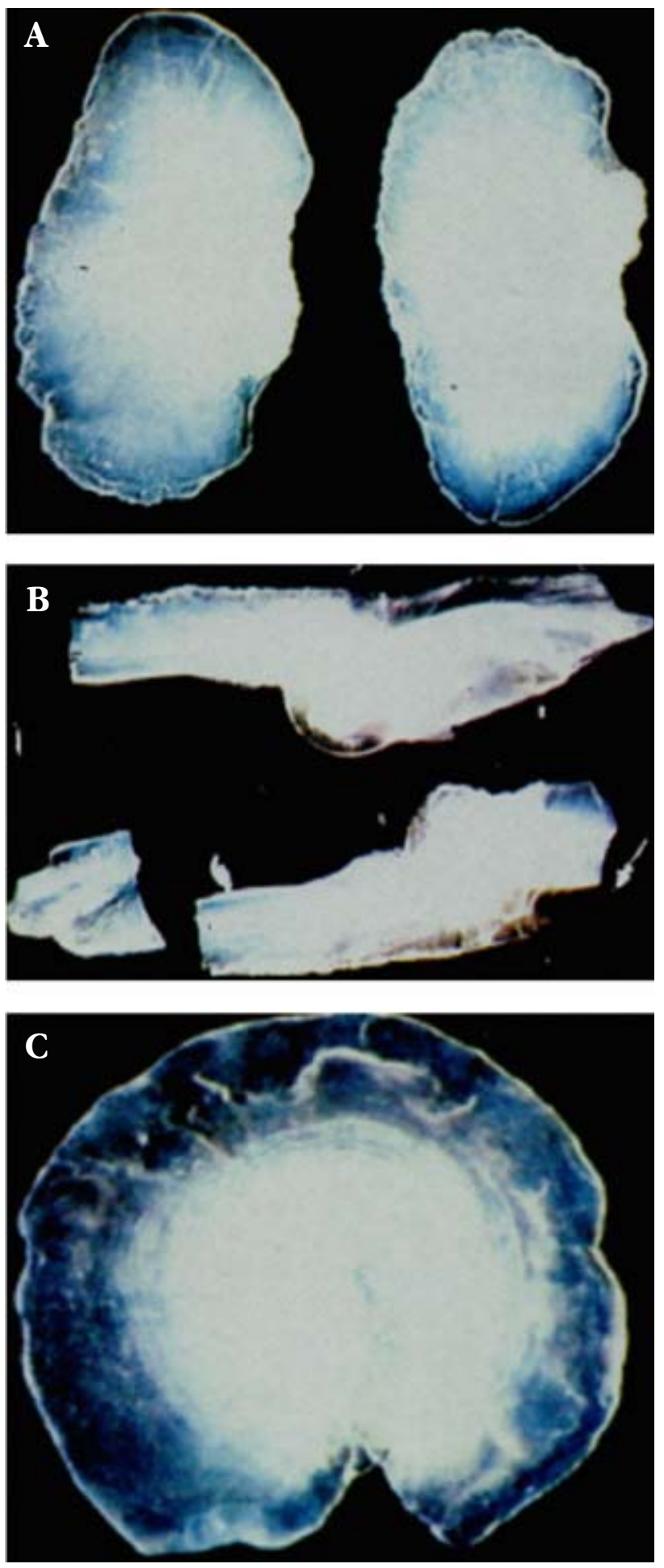

Figura 1 - Fotos de otólitos de Hypophthalmus marginatus : (a)-otólito lapilus $4,2 \times 2,2 \mathrm{~mm}$.

(b)-otólito sagita 2,4 $\mathrm{x}$ de comp.

(c)-otólito asteriscus $2,4 \mathrm{~mm}$ de diâmetro 
tratamento químico, essa estrutura apresentou anéis bem nítidos. Deve-se ressaltar que a nitidez sofre influência da iluminação, havendo relação entre a nitidez dos anéis a tonalidade e intensidade da luz utilizada. Os resultados das leituras foram comparados entre si, notando-se que a partir da segunda leitura se estabilizaram os critérios de interpretação (Tabela 2).

Tabela 1 - Total de exemplares de mapará, H. marginatus, por mês e local de procedência.

\begin{tabular}{lccccc}
\hline \hline Mês & \multicolumn{2}{c}{ Local } & Machos & Fêmeas & Indeterminado Total \\
\hline Dez/96 & Manaquiri & 00 & 05 & 09 & 14 \\
Jan/97 & Janauacá & 10 & 10 & 46 & 66 \\
Fev/97 & Janauacá & 07 & 15 & 09 & 31 \\
Mar/97 & Janauacá & 05 & 05 & 18 & 28 \\
Abr/97 & Manaquiri & 00 & 07 & 22 & 29 \\
Mai/97 & Janauacá & 10 & 09 & 11 & 30 \\
Jun/97 & Janauacá & 03 & 06 & 02 & 11 \\
Jul/97 & Janauacá & 04 & 07 & 13 & 24 \\
Ago/97 & Janauacá & 03 & 09 & 23 & 35 \\
\hline Total & & 42 & 73 & 153 & 268 \\
\hline \hline
\end{tabular}

Tabela 2 - Leituras comparativas efetuadas para estabilização de critérios de interpretação de anéis etários nos otólitos asteriscus de $H$. marginatus.

\begin{tabular}{|c|c|c|c|c|c|c|}
\hline \multicolumn{7}{|c|}{ 1a leitura } \\
\hline No anéis & 1 & 2 & 3 & 4 & 5 & 6 \\
\hline 1 & & & & & & \\
\hline 2 & & & 1 & 5 & 1 & 1 \\
\hline 萢 3 & & & & & 1 & \\
\hline ง 4 & & & 1 & & & \\
\hline 5 & & & & 1 & & \\
\hline 6 & & & & 2 & & 2 \\
\hline
\end{tabular}

\begin{tabular}{|c|c|c|c|c|c|c|}
\hline \multicolumn{7}{|c|}{ 1a leitura } \\
\hline No anéis & 1 & 2 & 3 & 4 & 5 & 6 \\
\hline \multicolumn{7}{|l|}{1} \\
\hline 뜰 2 & & & 1 & 5 & 1 & 1 \\
\hline 矛 3 & & & & & 1 & \\
\hline กี 4 & & & 1 & & & \\
\hline 5 & & & & 1 & & \\
\hline 6 & & & & 2 & & 2 \\
\hline \multicolumn{7}{|c|}{ 2a leitura } \\
\hline No anéis & 1 & 2 & 3 & 4 & 5 & 6 \\
\hline 1 & & 1 & & & & \\
\hline 롤 2 & 1 & 3 & & & & \\
\hline 氖 3 & & & 1 & & & \\
\hline ণึ 4 & & & 2 & 3 & 5 & 1 \\
\hline 5 & & & & 3 & 3 & \\
\hline 6 & & & & & & 2 \\
\hline
\end{tabular}

\section{Descrição do padrão ótico observado e morfometria.}

O asteriscus apresenta anéis mais nítidos quando se aplica luz incidente lateral. Apresentou um padrão regular até o penúltimo anel, seguindo do ultimo até a borda com padrões diferenciados, devido a dificuldade de discriminar as marcas principais das falsas em anéis próximos da borda do otólito. A explicabilidade do seu tamanho pela variação do comprimento furcal foi baixa (31,2\%), embora estatisticamente significativa $\left(\mathrm{F}_{1,184}=83,779 ; \mathrm{P}>0,01\right)$, o que indica que é inadequada para aplicação do retrocálculo. A plotagem da reta obtida com os parâmetros estimados na regressão, apresenta grande dispersão dos dados, e falta de ajuste ao modelo para otólitos de menor tamanho (Figura 2).

\section{Validação}

Observou-se que há dois períodos com menor incremento marginal relativo (Figura 3), o primeiro em meados da enchente (Jan-Fev), o segundo no inicio da cheia (Mai-Jun). Os menores incrementos marginais relativos ocorreram em fevereiro e em junho.

\section{Determinação dos Parâmetros Populacionais.}

Os parâmetros de crescimento foram estimados apenas com os dados de leitura direta, não sendo utilizado o retrocálculo, devido a dispersão dos dados ter sido elevada.

Na tabela3, podem ser observados os valores dos parâmetros de crescimento obtidos neste trabalho utilizando leitura direta dos anéis de crescimento nos otólitos, sendo a curva de crescimento plotada na Figura 4. A esperança de vida $\left(\mathrm{A}_{0.95}\right)$, foi estimada em 5,42 anos e a mortalidade natural (M) em 0,55.

\section{DISCUSSÃO}

Oconhecimento do ritmo de crescimento em peixes nas regiões alagáveis daAmazônia Central éimportante para viabilizar estimativas mais precisas do potencial sustentável de produção pesqueira nesta região (Fabré \& Saint Paul, 1998). Tais informações também podem ser aplicadas em piscicultura para determinar a potencialidade e a possibilidade lucrativa da criação de determinada espécie, pois esses conhecimentos nos fornecem parâmetros de referência para avaliar o rendimento da produção (Villacorta-Correa, 1997).

Estudos de determinação de idade em peixes de regiões temperadas, têm indicado que as marcas de crescimento (anéis etários), são formados de acordo com as estações do ano devido basicamente às variações da temperatura do ambiente (Casselman, 1983). Na Amazônia, devido a pequena variação térmica ao longo do ano, acreditava-se que as marcas de crescimento não apareceriam nas estruturas calcificadas, portanto, a determinação de idade por este método não seria útil (Worthmam, 1983; Sparre \& Venema, 1995). Contudo, trabalhos na região comprovam a existência de marcas bem definidas em diversas estruturas calcificadas em peixes, como 


\section{ACTA \\ AMAZONICA}

DETERMINAÇÃO DE IDADE E CRESCIMENTO DO MAPARÁ (HYPOPHTHALMUS MARGINATUS) NA AMAZÔNIA CENTRAL. escamas, otólitos, espinhos e vértebras (Villacorta-Correa, 1987; Welcome, 1992; Oliveira,1997; Fabré \& Saint Paul, 1998; PerezLozano, 1999; Vieira, 1999). A determinação da idade do mapará daAmazônia Central, realizada no presente trabalho, e os trabalhos já citados confirmam que marcas nas estruturas calcificadas podem indicar o ritmo de crescimento em peixes na Amazônia.

O mapará é um peixe que sofre um esforço de pesca direcionado, sendo comercializado pelos frigoríficos da região que exportam seu filé beneficiado para o sudeste do Brasil e para alguns países europeus e América do Norte. No estado do Amazonas a população local não consome este Siluriforme devido a tabus culturais sobre os peixes de couro ou peixes-lisos (Smith, 1979; Batista et al., 1998). Apesar de espécies do gênero Hypophthalmus representarem importantes recursos pesqueiros na economia regional (Mencia-Morales et al., 1976; Isaac \& Bathem, 1995), poucos estudos foram realizados (Marlier,1967; Silva, 1972; Carvalho, 1980; Alcântara-Neto, 1994), e nenhum relacionadoà determinação de idade usando estruturas calcificadas.

Apesar de que para alguns Siluriformes, como o surubim (Panfilli, 1993) e a piracatinga (Perez-Lozano, 1999), as vértebras tenham sido indicadas como melhores para determinação de idade, os otólitos têm sido apontados por muitos autores como as estruturas calcificadas que melhor reflete a idade dos peixes (William \& Bedford, 1974; Hetch, 1980; Baker \& Timmons, 1991), sendo o mesmo observado no presente estudo e para outros Siluroides da Amazônia, como a dourada Brachyplatystoma flavicans (Rego et al 1998; Alfonso \& Fabré, 2002).

A coleta e a forma pela qual a estrutura é preparada são etapas importantes antes de se realizar qualquer estudo sobre idade e crescimento. A estrutura elegida e seu método de tratamento devem propiciar a melhor visualização do padrão de marcação, por outro lado a estrutura deve ser de fácil coleta e manuseio, para facilitar o trabalho em larga escala. O mapará, ao contrario de outros Siluriformes, possui uma caixa craniana de ossos bem moles o que facilita a coleta dos otólitos.

Na maioria dos trabalhos realizados com otólitos, cerca de $60 \%$ dos pesquisadores escolheram o par sagitta para realizar estudos de microestruturas (Villacorta-Correa, 1997), por serem maiores, de fácil manuseio e apresentarem incrementos mais largos (Secor et al., 1992). No mapará, o par escolhido foi o asteriscus, que não é o maior, mas foi o que apresentou formato mais homogêneo e anéis mais nítidos, além de serem fáceis de coletar e não requererem nenhum outro procedimento, como polimento ou corte transversal.

Como a maioria dos otólitos não têm uma morfologia padrão, é recomendável que se defina o melhor ângulo para serem efetuadas as leituras e que este seja obedecido até o final das mesmas (Campana, 1992). O melhor resultado obtido foi similar ao indicado porVillacorta-Correa, (1997), sendo que a posição padrão de leitura dos anéis da estrutura corresponde a uma região com espessura intermediária dentre as disponíveis no otólito.

Não foi necessário utilizar nenhuma técnica de clarificação nos otólitos de mapará para visualização de marcas de crescimento. Há indícios de que, o par lapillus apresenta anéis etários que podem ser visualizados com técnicas de corte e de tratamento químico, devido a sua espessura. A nitidez dos otólitos asteriscus, aliado a facilidade na sua manipulação não justifica o trabalho extra com os mesmos.
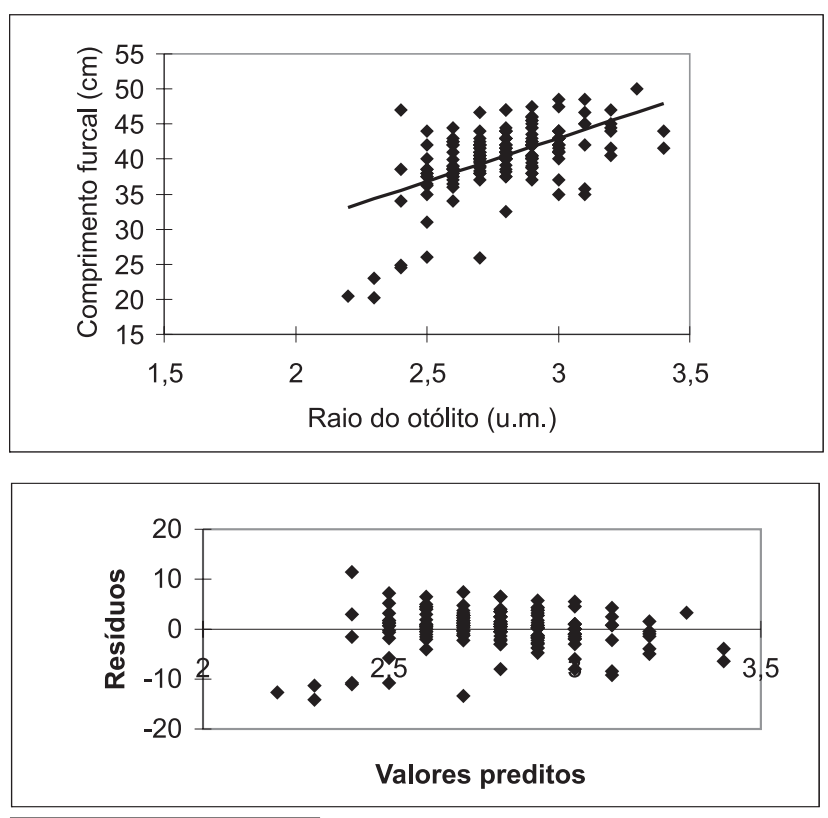

Figura 2 - Relação entre o comprimento furcal / raio do otólito de Hypophthalmus marginatus (acima) e as respectivas distribuições de resíduos (abaixo).

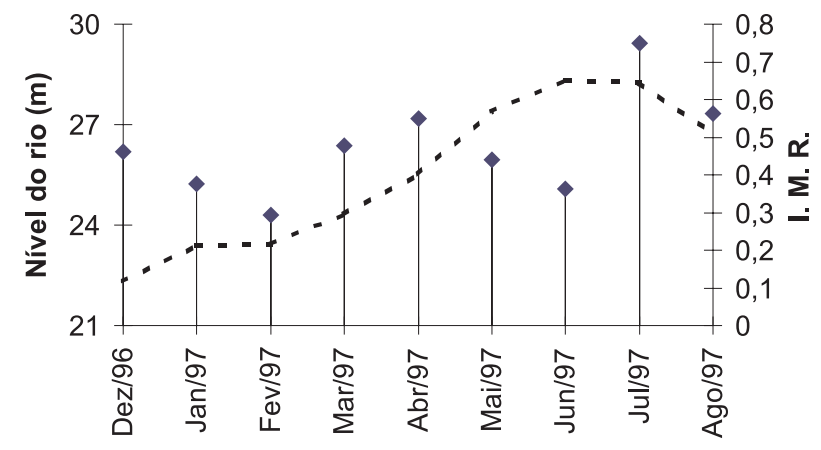

Nível do rio -.-.-.

Figura 3 - Valores médios mensais do incremento marginal relativo(IMR) das marcas, nos otólitos e variação do nível do rio, adquiridos na ANEEL, de dezembro/96 a agosto/97.E

Tabela 3 - Parâmetros populacionais estimados para Hypophthalmus marginatus

\begin{tabular}{lc}
\hline \hline Parâmetros & H marginatus \\
\hline Taxa de crescimento (k) & 0,55 ano-1 \\
Comprimento assintótico ( Lo) & $52,63 \mathrm{~cm}$ \\
Esperança de vida (A95) & 5,4 anos \\
Taxa de mortalidade natural (M) & 0,55 \\
\hline \hline
\end{tabular}




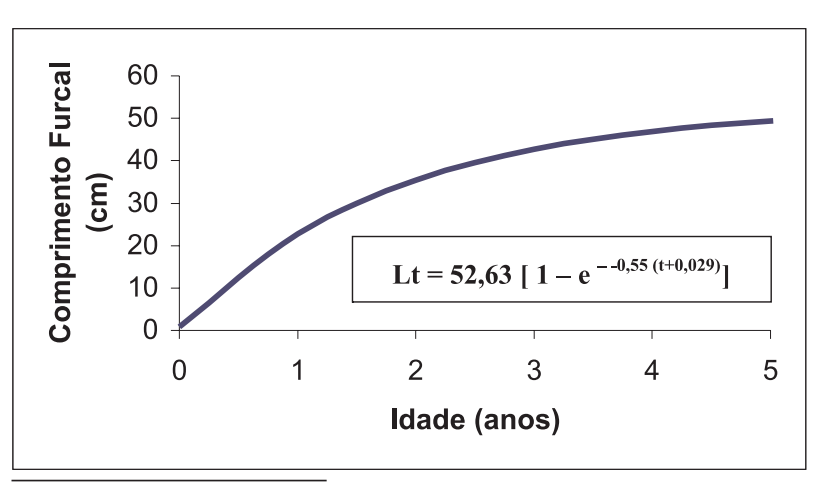

Figura 4 - Curva de crescimento estimada para $H$. marginatus, com indicação da equação de crescimento para a espécie.

Segundo Panfili (1993) é necessário caracterizar as marcas de crescimento nas estruturas calcificadas elegidas, como um estudo preliminar para determinar a idade e crescimento em peixes tropicais, padronizando as marcas falsas das verdadeiras. Apesar disso, no período compreendido entre 1907 e 1960 apenas 15 de 500 trabalhos consultados tinham algum estudo de validação (Walter, 1997). Isto é grave pois é a validação que determina a relação entre a contagem de anéis e o tempo cronológico.

O número de anéis de crescimento apenas pode ser relacionado com idade se há identificação das marcas verdadeiras e se a cronologia do aparecimento das marcas é estabelecida. Nos otólitos de Colossoma macropomum, os anéis puderam ser claramente separados das marcas acessórias, sendo que os principais entraves foram da definição do primeiro anel e as extensas zonas opacas e hialinas que dificultaram a observação dos anéis em alguns otólitos (Villacorta-Correa, 1997). Nos otólitos de mapará, esses anéis de crescimento foram separados dos anéis falsos, visto que estes não apresentavam continuidade nem padrão. Além dos entraves indicados, notou-se dificuldade em visualizar anéis muito próximos à borda.

O método do incremento marginal é prático e tem sido o mais utilizado para validar marcas indicativas de crescimento nos estudos recentes de determinação de idade realizados na Amazônia (Loubens \& Panfili, 1992; Villacorta-Correa, 1997; Oliveira, 1997;; Oliveira,1997; Fabré \& Saint-Paul, 1998; Perez Lozano, 1999; Vieira, 1999).

No presente trabalho, a análise do crescimento sazonal pelo método do incremento marginal indicou a formação de duas marcas principais por ano nos otólitos do mapará da Amazônia Central. A formação de dois anéis por ano, também foram registrados em peixes tropicais por Moreau (1975; 1977), Villacorta-Correa (1987), Oliveira (1997), Vieira, (1999), Perez-Lozano, (1999) e Alonso , (2002.) porém várias podem ser suas causas de formação. Mesmo não podendo afirmar com precisão os fatores ambientais ou biológicos que influenciam na formação das marcas nas estruturas calcificadas dos peixes amazônicos, um padrão sazonal pode ser reconhecido (Oliveira, 1997).

Para o mapará (H. marginatus) foi registrado que anéis são formados em fevereiro (na enchente) e em junho (na cheia ou início da vazante). A primeira marca (Fev-Mar), pode ser atribuída ao estresse ocasionado pela migração reprodutiva e conseqüente desova, que segundo Carvalho (1980), ocorre em abril. Segundo Vieira (1999), a migração reprodutiva causa estresse no peixe, ocasionando uma diminuição no ritmo de crescimento. Asegunda marca (Jun-Jul), ocorreu no pico de cheia, um período propicio para a diminuição no ritmo de crescimento da espécie, já que a inundação das planícies marginais e lagos, causa dispersão no seu principal alimento, o zooplâncton e microcrustáceos, que apresentam maior densidade na época de seca em lagos da Amazônia (Brandorff, 1978).

Para calcular o comprimento em idades passadas utilizando anéis etários de estruturas calcificadas (método do retrocálculo) é necessário haver um modelo que expresse o comprimento na captura como uma função do raio da estrutura. Neste trabalho, embora o modelo linear seja adequado para a maior parte dos dados na relação do comprimento furcal versus o raio do otólito, o ajuste foi fraco quando o raio do otólito foi menor de $2,5 \mathrm{~mm}$, em peixes com comprimento inferior a 30 $\mathrm{cm}$, tornando inviável o método do retrocálculo.

Segundo Beverton \& Holt (1957), suprimento alimentar e densidade populacional, são fatores principais que afetam o L¥ , já o ké determinado principalmente por causas fisiológicas. $\mathrm{O}$ valor de k encontrado neste trabalho para $H$. marginatus, indica que esta espécie possui uma elevada taxa de crescimento $(\mathrm{k}=0,55)$ comparado com outras espécies de peixe da Amazônia (Tabela 4).

Tabela 4 - Coeficientes de crescimento estimados em trabalhos realizados em espécies de peixe da Amazônia Central comparativamente com o estimado para o mapará

\begin{tabular}{lcc}
\hline \hline Estudo & Espécie & $\mathbf{k}$ \\
\hline Ruffino \& Isaac, 1995; & Prochilodus nigricans & 0,$50 ;$ \\
Oliveira, 1997 & Colossoma macropomum & 0,46 \\
Ruffino \& Isaac, 1995; & 0,15 \\
Villacorta-Correa,1997 & Schyzodon fasciatus & 0,52 \\
Ruffino \& Isaac, 1995 & Semaprochilodus insignis & 0,50 \\
Vieira, 1999 & Calophysus macropterus & 0,41 \\
Perez Lozano, 1999 & Hypophthalmus marginatus & 0,55 \\
\hline Cutrim \& Batista, este trabalho & & \\
\hline \hline
\end{tabular}

Tabela 5 - Comparações entre a mortalidade natural encontradas em outros estudos realizados em peixes da região

\begin{tabular}{lcc}
\hline \hline Autor & Espécie & M \\
\hline Oliveira, 1997 & Prochilodus nigricans & 0,47 \\
Perez Lozano, 1999 & Calophysus macropterus & 0,42 \\
Vieira, 1999 & Semaprochilodus insignis & 0,53 \\
Cutrim \& Batista, este trabalho & Hypophthalmus marginatus & 0,55 \\
\hline \hline
\end{tabular}




\section{ACTA \\ AMAZONICA}

Já a mortalidade natural estimada de 0,55 está ao mesmo nível das estimativas já efetuadas na região para outras espécies explotadas (Tabela 5).

Portanto, sendo espécie zooplanctófaga, apresentando ritmo de crescimento rápido, alta mortalidade natural e baixa esperança de vida, pode ser considerada uma espécie restrategista.

O mapará é um recurso amplamente explotado. Deve-se porém efetuar o monitoramento da exploração e das respostas que o estoque apresentará frente a variação do mesmo, aspecto fundamental para a sustentabilidade da atividade e perpetuação do recurso.

\section{CONCLUSÕES}

- Para a espécie estudada, o mapará (H. marginatus), o par de otólito asteriscus, demonstrou ser o mais adequado para determinação de idade.

- A variabilidade entre o comprimento do peixe e o raio do otólito elegido, sugere que não se utilize o retrocálculo.

- Há um aumento no comprimento dos otólitos a medida que aumenta o tamanho do peixe, tornando útil os otólitos para determinação de idade.

- O crescimento do mapará em comprimento foi descrito pela seguinte equação:

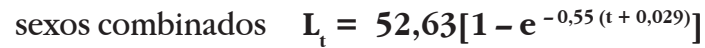

- O mapará tem um ciclo de vida curto sendo sua esperança de vida de 5,4 anos, o crescimento rápido e a mortalidade natural alta $(\mathrm{M}=0,55)$.

\section{BIBLIOGRAFIA CITADA}

Alcântara Neto, C.P. 1994. Ecologia da pesca dos maparás, Hypophthalmus spp. (Siluriformes, Hypophthalmidae), no lago Grande de Monte Alegre, Baixo Amazonas, Pará. Dissertação de Mestrado. Universidade Federal do Pará/ Museu Paraense Emílio Goeldi. Belém, Pará. 141pp.

Baker, T. T.; Timmons, L. S. 1991. Precision of ages from bony structures of artic charn (Salvelinus alpinus) form the Wood River System, Alaska. Can. J. Fish. Aquat. Sci., 48:1007-1014.

Batista, V.S.; Inhamuns, A.J.; Freitas, C.E.C. , Freire-Brasil, D. 1998. Characterization of the fishery in river communities in the lowSolimões/high-Amazon region. Fish. Manage. Ecol., 5:419-435.

Beverton, R. J.; Holt, S. J. 1957. A review of the life spans and mortality rates in nature, and their relation to growth and other physiological characteristics. Ciba Foundation Colloquium on Ageing, 5:142-177.

Brandorff, G. O; Andrade, E. R. 1978 The relationship between the water level of the Amazon River and fate of the zooplakton population in lago Jacaretinga, a várzea lake in Central Amazon. Stud. Neotrop. Fauna Environ., 13:63-70.

Campana SE. 1992. Measurement and interpretation of the microstructure of fish otoliths. In: Stevenson D. K., Campana S. E. (Eds.). Otolith microstructure examination and analysis. Can. Spec. Publ. Fish. Aquat. Sci., 117:. 59-71
Carvalho, F. M. 1980 a. Alimentação de Mapará (Hypophthalmus edentatus Spix 1829) do Lago Castanho, Amazonas (Siluriformes, Hypophthalmidae). Acta Amazonica, 10(3):545-555.

Carvalho, F. M. 1980 b. Composição química e reprodução (Hypophthalmus edentatus, Spix 1829) do Lago Castanho, Amazonas (Siluriformes, Hypophthalmidae). Acta Amazonica., 10(2):379-389.

Casselman, J.M. 1983. Age and growth assessment of fish from their calcified structures - tecniques and tools. In: Prince, E.D.; Pulos, L.M. (eds.). Proceedings of the International Workshop on Age Determination of Oceanic Pelagic Fishes: Tunas, Billfishes, and Sharks. NOAA Technical Report NMFS, $8: 1-17$.

Casselman, J. M. 1983. Age and growth assessment of fish from their calcified structures - techniques and tools. In: Prince E. D.; Pulos L. M. (Eds.) Proceedings of the International Workshop on Age Determination of Oceanic pelagic Fishes: Tuna, Billfishes, and Sharks. NOAA Technical Report:29-33.

Cutrim, L. 1997. Análise de estruturas rígidas de aposição para determinação de idade do mapará (Hypophthalmus spp.) na Amazônia Central. Anais VI Semana de Biologia. UA/ CNPq.:20.

Fabré, N.N.; Saint-Paul, U. 1998. Annulus formation on scales and seazonal growth of the Central Amazonian Anostomid Schizodon fasciatus (Osteichthyes, Characoidei). J. Fish Biol., 53:1-11.

Hetch, T. 1980. Age, growth, reproduction and mortality of the butter-catfish Eutropius depresirostris (Schilbeidae) : Pisces) in the Luphephe Uwanedzi Impoundment, Venda (South Africa).J. Limnol. Soc. Sth. Afr., 6(1):39-45.

Isaac, V. J.; Barthem, R. B. 1995. Os Recursos Pesqueiros da Amazônia Brasileira. Bol. Mus. Para. Emílio Goeldi, ser. Zool., 11(2):151-194.

Lai, H. L. 1987. Age determination of pacific cod Gadus macrocephalus using five ageing methods. Fish. Bull., 85:713-723.

Loubens , G.; Panfili, J. 1992. Estimation de I' àge individuel del Prochilodus nigricans (Teleosteo, Prochilodontidae) dans le Béni (Bolivie) protocole d'étude et aplication. Aquat. Living Resour., 5:41-56.

Marlier, G. 1967. Ecological studies on some lakes of the Amazon Valley. Amazoniana, 1(2):91-115.

Mencia Morales, F; Nunes, P. C. M.; Machado, J. C.; Amado, A. L. ; Souza, R. R.; Leite, L. W. N. 1976. Avaliação das industrias pesqueiras dos estados do Amazonas, Pará e Maranhão: capacidade produção e mercado. Brasília, Programa de Pesquisa e desenvolvimento pesqueiro no Brasil. PNUD/FAO - Ministério da Agricultura, SUDEPE, Brasília, Brasil. 68 p.

Merona, B. 1993. Pesca e ecologia dos recursos aquáticos na Amazônia. In: Furtado L.; Leitão W.; Melo, A. F (Org.) Povos das Águas: realidade e perspectivas na Amazônia., Museu Paraense Emílio Goeldi, Belém, Pará. p.159-186. 
Moreau, J. 1975. Essai d'interpretation des annuli observes sur les écailes de Tilapia nilotica et Tilapia rendali des lacs des hauts plateaux Malgaches. II. Observations sur Tilapia rendali au la Alaotra. Ann. Hydrobiol., 6(2): 93-101.

Moreau, J. 1977. Essai d'interpretation des annuli observes sur les écailes de Tilapia nilotica et Tilapia rendali des lacs des hauts plateaux Malgaches. III. Observations sur Tilapia rendali au la Alaotra. Ann. Hydrobiol., 8(3): 363-373.

Oliveira, B. I. 1997. Determinação da idade e aspectos da dinâmica populacional do curimatã Prochilodus nigricans (Pisces; Prochilodontidae) da Amazônia Central. Dissertação de mestrado apresentada ao curso de PósGraduação em Biologia de Água Doce e Pesca Interior, INPA/ FUA, Manaus, Amazonas. 90 pp.

Panfili, J. 1993. Estimation de age individuel des poissons: methodologies et applications á des populations naturelles tropicales et tempérées. Diplome de Doctorat, Université de Monpellier France. 456pp.

Pauly, D. 1983. Some simple methods for the assessment of tropical fish stocks. FAO Fish. Tech. Pap., 234:252p.

Peréz Lózano, A. 1999. Determinação de idade e crescimento da piracatinga Calophysus macropterus, Lichtenstein, 1819 (Pisces Pimelodidae), na Amazônia Central. Dissertação de Mestrado. INPA/FUA, Manaus, Amazonas: 87 pp.

Rego, H.V.; Fabré, N.N.; Lozano, A.P. 1998. Estruturas calcificadas de dourada (Brachyplatystoma flavicans) para determinação da idade na Amazônia Central. Bol. Mus. Para. Emílio Goeldi, Ser. Zool. 14 (2): 143-173.

Ruffino, M.L. , Isaac, V.J. 1995. Life cycle and biological parameter of several brazilian amazon fish species NAGA. The ICLARM Quartely, 4:41-45.

Secor, D. H. ; Dean, J. M.; Laban, E. H. 1992. Otolith removal and preparation for microstructural examination. In: Stevenson, D. K; Campana, S. E. (Eds). Otolith microstructure examination and analysis. Can. Spec. Publ. Fish. Aquatic. Sci., 117. p.19-57.

Silva, A. B. 1972. Relatório da viagem ao Estado do Pará, para captura de espécies de peixes: mapará (Hypophthalmus edentatus) e piramutaba (Brachyplatystoma vaillantti). Convênio DPAN, Fortaleza, 11 pp.
Smith, N. J. H. 1979. A pesca no rio Amazonas. CNPq - INPA, Manaus-AM. 154pp.

Sparre, P.; Venema, S. C. 1995. Introducción a la evolución de recursos pesqueros tropicales. Parte 1. Manual. FAO Doc. Tecn. Pesca, Rome, 306/1, Rev. 1. 440pp.

Vieira, E. F. 1999. Determinação da idade e crescimento do jaraqui escama grossa (Semaprochilodus insignis), na Amazônia Central. Dissertação de Mestrado. INPA/FUA, Manaus. 86pp.

Vieira, S.; Hoffmann, R. 1989. Estatística experimental. Editora Atlas, São Paulo, Brasil. 179pp.

Villacorta-Correa, M. A. 1987. Crescimento do matrinxã, Brycon cephalus (Günter, 1969) (Teleostei, Characidae) no baixo rio Negro, seus afluentes e no baixo Solimões. Dissertação de Mestrado. INPA/FUA, Manaus, Amazonas. 124pp.

Villacorta-Correa, M. A. 1997. Estudo de idade e crescimento do tambaqui Colossoma macropomum (Characiformes, Characidae) no Amazonas Central, pela análise de marcas sazonais nas estruturas mineralizadas e microestruturas nos otólitos. Tese de Doutorado. INPA/FUA, Manaus, Amazonas. 217pp.

Walter, T. 1997. Curva de crescimento aplicada para organismos aquáticos. Um estudo de caso para a toninha Pontoporia balinvillei (Cetacea Pontoporiidae) do extremo sul do Brasil. Monografia de bacharelado em Oceanografia FURG, Rio Grande, Brasil. 101pp.

Welcomme, R. L. 1992. Pesca fluvial. FAO Doc. Tecn. Pesca 262. Roma. 303 pp.

William, T.; Bedford, B. C. 1974. The use of otolith for age determination. In: Bagenal, T. B. (ed.), Ageing of Fish. Proceedings of the International Symposium. Unwin Brothers Limited, The Gresham Press, Surrey, England. p.114-123.

Worthmann, H. O. W. 1983. A comparative study of the growth of the post larval and juvenile pescadas Plagioscion squamosissimus (Heckel) and $P$. monti (Soares) in a White lake of the Central Amazon. Amazoniana, 7(4):465-477.

RECEBIDO EM 24/08/2003

ACEITO EM 20/01/2005 